\title{
Jahn-Teller reconstructed surface of the doped manganites shown by means of surface-enhanced Raman spectroscopy
}

\author{
S. Merten, ${ }^{*}$ V. Bruchmann-Bamberg, B. Damaschke, K. Samwer, and V. Moshnyaga \\ I. Physikalisches Institut, Georg-August-Universität Göttingen, Friedrich-Hund-Platz 1, D-37077 Göttingen, Germany
}

(Received 20 November 2018; revised manuscript received 30 April 2019; published 28 June 2019)

\begin{abstract}
We report direct evidence of the theoretically predicted electron-rich surface of doped perovskite manganites $\mathrm{La}_{0.7} A_{0.3} \mathrm{MnO}_{3}(A=\mathrm{Ca}, \mathrm{Sr})$ by means of surface-enhanced Raman spectroscopy. The required Au nanoparticles were grown on top of thin manganite films by the metalorganic aerosol deposition technique, which provides a stable oxygen atmosphere and prevents deoxygenation effects. The acquired surface-enhanced Raman spectra of rhombohedral $\mathrm{La}_{0.7} \mathrm{Sr}_{0.3} \mathrm{MnO}_{3}$ thin films reveal the symmetry-forbidden Jahn-Teller stretching modes demonstrating the predicted symmetry breaking at the surface and fallback into the orthorhombic (Pnma) structure. This results in a unique Jahn-Teller reconstructed surface structure, which limits the capabilities of mixed-valence manganites in spintronic applications, but will be highly favorable for catalytic reactions.
\end{abstract}

DOI: 10.1103/PhysRevMaterials.3.060401

\section{INTRODUCTION}

Mixed-valence perovskite manganites, such as $\mathrm{La}_{1-x} A_{x} \mathrm{MnO}_{3}(A=\mathrm{Sr}, \mathrm{Ca})$, possess strong electron-spinlattice correlations [1,2], which result in many fundamentally interesting and useful electronic properties. Some of them, such as a high degree of spin polarization [3] and a high catalytic activity [4], make manganites promising materials for many future applications, such as tunneling magnetoresistance (TMR) devices [5] or solid oxide fuel cells [6]. Therefore, a detailed knowledge of the surface structure is of crucial importance, as the electronic surface structure determines the catalytic and fuel cell performance as well as the spin polarization at the TMR interface.

Numerous theoretical studies have aimed at the understanding of the differently oriented $\mathrm{LaMnO}_{3}$ surfaces [7,8], the (001) surface of $\mathrm{CaMnO}_{3}[9,10]$, and the surface of the hole-doped $\mathrm{La}_{1-x} A_{x} \mathrm{MnO}_{3}(A=\mathrm{Ca}, \mathrm{Sr})$ [11-14]. The alterations at the surface compared to the bulk structure range from large surface rumpling [7,8,13-15], over a spin flip in the surface layer $[8,12]$ to a charge transfer from the bulk to the surface $[11,13,14]$. The latter results in the formation of a nonmagnetic, most likely canted antiferromagnetic, insulating $\mathrm{Mn}^{3+}$-rich layer at the surface. Coaxial impact-collision ion scattering spectroscopy on $\mathrm{La}_{0.7} \mathrm{Sr}_{0.3} \mathrm{MnO}_{3}$ revealed a $\mathrm{MnO}_{2}$-terminated (001) surface [16]. Photoemission studies of $\mathrm{La}_{0.7} \mathrm{Ca}_{0.3} \mathrm{MnO}_{3}$ and $\mathrm{La}_{0 .} \mathrm{Ca}_{0.1} \mathrm{MnO}_{3}$ showed further that the surface termination depends also on the doping, changing from a $\mathrm{MnO}_{2}$ to a (La,Ca)O termination with decreasing $x$ [17]. A nonmagnetic and insulating layer at the manganite

\footnotetext{
*Corresponding author: smerten@gwdg.de

Published by the American Physical Society under the terms of the Creative Commons Attribution 4.0 International license. Further distribution of this work must maintain attribution to the author(s) and the published article's title, journal citation, and DOI.
}

surface was observed by conducting atomic force microscopy $[18,19]$ in $\mathrm{La}_{0.7} \mathrm{Ca}_{0.3} \mathrm{MnO}_{3}$ and by $\mathrm{x}$-ray magnetic circular dichroism (XMCD) [20] as well as by $\mathrm{X}$-ray resonant magnetic scattering (XRMS) $[21,22]$ on $\mathrm{La}_{0.7} \mathrm{Sr}_{0.3} \mathrm{MnO}_{3}$ and the layered compound $\mathrm{La}_{2-2 x} \mathrm{Sr}_{1+2 x} \mathrm{Mn}_{2} \mathrm{O}_{7}$. Segregation effects, which are well known in manganite thin films [23,24], lower the effective doping at the surface and can result in the reconstruction of the surface layer, forming a Ruddleson-Popperlike phase [25] or in a change of the manganese valence, as shown in $\mathrm{La}_{1-x} \mathrm{~Pb}_{x} \mathrm{MnO}_{3}$ [26]. In the latter case, the valence change was attributed to the possible higher oxidation states of $\mathrm{Pb}$, compared to $\mathrm{Sr}$ or $\mathrm{Ca}$. These extrinsic segregation effects are related to oxygen deficiencies and thus depend on the growth conditions, i.e., temperature and oxygen partial pressure $[27,28]$. They provide a serious obstacle for the study of manganite surfaces, since they alter the surface chemistry significantly and, sometimes, in an irreversible way. As a result, despite an enormous experimental effort, the theoretical predictions have yet to be proven and a mature picture of the surface nature is missing.

Over the last 20 years, Raman spectroscopy was established as a powerful tool to study correlated materials [29], including mixed-valence manganites [30-32]. Numerous Raman studies engaged in the correlation of the observed Raman modes to the presence of Jahn-Teller (JT) distortions in the manganite system $[33,34]$ as well as in their relation to the metal-insulator and ferromagnetic-paramagnetic phase transition [31,33]. In the Raman spectra, these JT distortions, which are inherent for the orthorhombic (Pnma) structure of the lightly doped manganites $(x<0.5)$, are represented by two high-frequency stretching modes at 490 and $611 \mathrm{~cm}^{-1}[31,33]$. In rhombohedral $\mathrm{La}_{0.7} \mathrm{Sr}_{0.3} \mathrm{MnO}_{3}$, in turn, these modes are forbidden [32,35,36], and instead the Raman spectra are dominated by a sharp bending mode at $442 \mathrm{~cm}^{-1}$. Therefore, the appearance of the JT modes can be used as a fingerprint of the lattice structure of the manganite film. Unique Raman techniques to probe the surface structure of manganites or other correlated oxides could be 
tip-enhanced (TERS) and/or surface-enhanced Raman spectroscopy (SERS), which both exploit the excitation of surface plasmons in metallic nanostructures to enhance the Raman signal from nearby adsorbates [37]. The strongly localized character of the surface plasmon, i.e., its electric field $E(r)_{\mathrm{SP}}$ scales with $1 / r^{3}$, limits the probing area down to a few nanometers $\left(d_{\mathrm{SP}} \approx 4-5 \mathrm{~nm}\right)$ [38,39], making the study of the manganite surface by means of Raman spectroscopy in principle possible. However, up to now only a few studies were performed on oxides, mostly focused on nanoparticles rather than thin films [40-42]. Moreover, to the best of our knowledge, only one TERS study on a strongly correlated material, the double perovskite $\mathrm{La}_{2} \mathrm{CoMnO}_{6}$ [43], was published, but no surface-oriented studies of the mixed-valence manganites, $\mathrm{La}_{1-x} A_{x} \mathrm{MnO}_{3}$, were reported up to now.

In this Rapid Communication, we present a SERS study on thin $\mathrm{La}_{0.7} \mathrm{Ca}_{0.3} \mathrm{MnO}_{3}$ (LCMO) and $\mathrm{La}_{0.7} \mathrm{Sr}_{0.3} \mathrm{MnO}_{3}$ (LSMO) films. While the surface Raman spectra of LCMO are enhanced but congruent, the surface of LSMO reveals the dominance of two Jahn-Teller-like Raman modes. This gives evidence for the presence of the cooperative JT effect due to the symmetry-breaking-induced electron enrichment at the LSMO surface.

\section{EXPERIMENTAL TECHNIQUES}

LSMO and LCMO films have been grown by a metalorganic aerosol deposition (MAD) technique [44] on $\mathrm{MgO}(100)$ substrates (Crystal $\mathrm{GmbH}$ ). Acetylacetonates of $\mathrm{La}, \mathrm{Sr}, \mathrm{Ca}$, and $\mathrm{Mn}$ were used as precursors. The films were grown at a substrate temperature $T_{\text {sub }}=950{ }^{\circ} \mathrm{C}$ with a growth rate $v=10 \mathrm{~nm} / \mathrm{min}$ and cooled down to room temperature in $15 \mathrm{~min}$. The gold nanoparticles (Au-NPs) were subsequently deposited by MAD at $T_{\text {sub }}=550-570^{\circ} \mathrm{C}$, using a gold(III) acetate precursor (Alfa Aesar, 99.9\%) dissolved in dimethylformamide to a concentration $c=0.02 \mathrm{M}$. The solution with a volume $V=1 \mathrm{ml}$ was sprayed onto the manganite film with a deposition rate $v=0.011 \mathrm{ml} / \mathrm{s}$. Finally, the prepared $\mathrm{Au}-\mathrm{NP} / \mathrm{LS}(\mathrm{C}) \mathrm{MO} / \mathrm{MgO}(100)$ samples were cooled down to room temperature in $10 \mathrm{~min}$. X-ray diffraction in $\Theta-2 \Theta$ Bragg-Brentano geometry with $\mathrm{Cu} K_{\alpha}$ radiation and smallangle $\mathrm{x}$-ray reflectivity measurements were performed to characterize the structure and thickness of the films. Magnetic (Magnetic Property Measurement System, Quantum Design) and electrical four-probe characterizations (Physical Property Measurement System, Quantum Design) were carried out for temperatures $T=5-400 \mathrm{~K}$. The morphology and size of the Au-NPs were characterized via scanning electron microscopy (SEM, Leo Supra 35). A four-lens optical reflection setup under normal incidence, combined with a UV-VIS spectrometer (Maya 2000Pro USB, Ocean Optics), was used to determine the wavelength of the surface plasmon resonance $\lambda_{\text {SPR }}$. The Raman spectra were acquired in a backscattering geometry with a confocal Raman microscope (LabRAM HR Evolution, Horiba Jobin Yvon) equipped with a thermoelectrically cooled charge-coupled device of $1024 \times 256$ pixels. A continuous-wave HeNe laser, $\lambda=632.8 \mathrm{~nm}$, with a spot size of $d<1 \mu \mathrm{m}$, was used and the laser power at the surface was kept at $P_{0}=0.65 \mathrm{~mW}$ during the measurement.

\section{RESULTS AND DISCUSSION}

Structural, electrical, and magnetic characterization of thin LCMO and LSMO films are shown in Figs. S1.1- S1.4 in the Supplemental Material (SM) SM I [45] demonstrating the high quality of our thin manganite films. The estimated pseudocubic lattice constants, $a_{\mathrm{LCMO}}=3.867 \AA$ and $a_{\mathrm{LSMO}}=3.882 \AA$, are very close to the corresponding bulk values $[46,47]$ indicating a strain-free state of the manganite films on $\mathrm{MgO}(100)$. Electrical and magnetic measurements reveal a coupled ferromagnetic-paramagnetic and metal-insulator transition, $T_{\mathrm{MI}}=T_{\mathrm{C}}=267 \mathrm{~K}$, for LCMO and a ferromagnetic-paramagnetic transition, $T_{\mathrm{C}}=364 \mathrm{~K}$, and metal-metal transition, $T_{\mathrm{MM}}=343 \mathrm{~K}$, for LSMO. After the deposition of the $\mathrm{Au}$ layer, the structural, electrical, and magnetic properties of our manganite films did not change. This is in line with density functional theory calculations [48] and x-ray photoemission spectroscopy measurements [49] showing no chemical interaction between an Au layer and the manganite surface. However, Brivio et al. observed a drastic decrease of the Curie temperature for ultrathin films with a thickness less than $8 \mathrm{~nm}$ [50]. They attributed the observed decrease of $T_{\mathrm{C}}$ by $\approx 150 \mathrm{~K}$ for a 4-nm-thick LSMO film to deoxygenation effects during the deposition of the $\mathrm{Au}$ layer. Oxygen vacancies, strongly affecting the properties of the manganites [51], are a well-known problem in common thin-film deposition techniques using ultrahigh vacuum, e.g., pulsed laser deposition. However, the high partial oxygen pressure, $p\left(\mathrm{O}_{2}\right) \approx 0.2$ bar, within the MAD prevents or at least minimizes the formation of oxygen vacancies during the deposition. This is supported by annealing experiments done on MAD-grown Ruddleson-Popper $\mathrm{SrO}\left(\mathrm{SrTiO}_{3}\right)_{n}$ heterostructures [52]. To verify the stability of our films with respect to deoxygenation effects caused by the Au deposition, we prepared an ultrathin LSMO film, $d=12$ u.c. $(\approx 4.6 \mathrm{~nm})$, on a $\mathrm{TiO}_{2}$-terminated $\mathrm{STO}(100)$ substrate. Since the thickness is much smaller than $8 \mathrm{~nm}$, one could expect a strong reduction of the $T_{\mathrm{C}}$ and $T_{\mathrm{MM}}$, because of deoxygenation. As one can see in Fig. 1, only minimal changes of the transition temperatures, $\Delta T_{\mathrm{C}}=3 \mathrm{~K}$ and $\Delta T_{\mathrm{MM}}=7 \mathrm{~K}$, occur, compared to the huge decrease in previous works $[50,53]$. Therefore, deoxygenation effects are negligible in our films. SEM images of the manganite surface show a homogeneous formation of $\mathrm{Au}-\mathrm{NPs}$ without any indication of clustering. Nevertheless, the particle size is distributed over a rather broad range with a mean particle size $d_{\mathrm{NP}}=15-16 \mathrm{~nm}$ [see Fig. 1(d)], as it is common for a self-aggregated growth of nanoparticles [54]. The reflectance of the studied Au-NP/manganite films shows a surface plasmon resonance (SPR) around $\lambda_{\mathrm{SPR}}=$ $592-606 \mathrm{~nm}$, indicated by the broad reflectance peak $[45,55]$ seen in Fig. 1(c).

In Fig. 2(a), the Raman spectra of thin LCMO films at room temperature are shown. A detailed explanation of the processing of the raw Raman spectra is given in SM II [45]. The spectra are dominated by a rotational mode at $228 \mathrm{~cm}^{-1}\left(A_{1 \mathrm{~g}}\right)$ and by the two broad JT stretching modes: antistretching at $499 \mathrm{~cm}^{-1}$ (as-JT, $A_{1 \mathrm{~g}}$ ) and stretching at $609 \mathrm{~cm}^{-1}$ (s-JT, $B_{2 \mathrm{~g}}$ ), respectively $[30,33,34]$. One can also see a small contribution of the bending mode at $438 \mathrm{~cm}^{-1}\left(B_{2 \mathrm{~g}}\right)$. The remaining modes at $343 \mathrm{~cm}^{-1}\left(B_{2 \mathrm{~g}}\right)$ and $438 \mathrm{~cm}^{-1}\left(A_{1 \mathrm{~g}}\right)$ can be also assigned to 


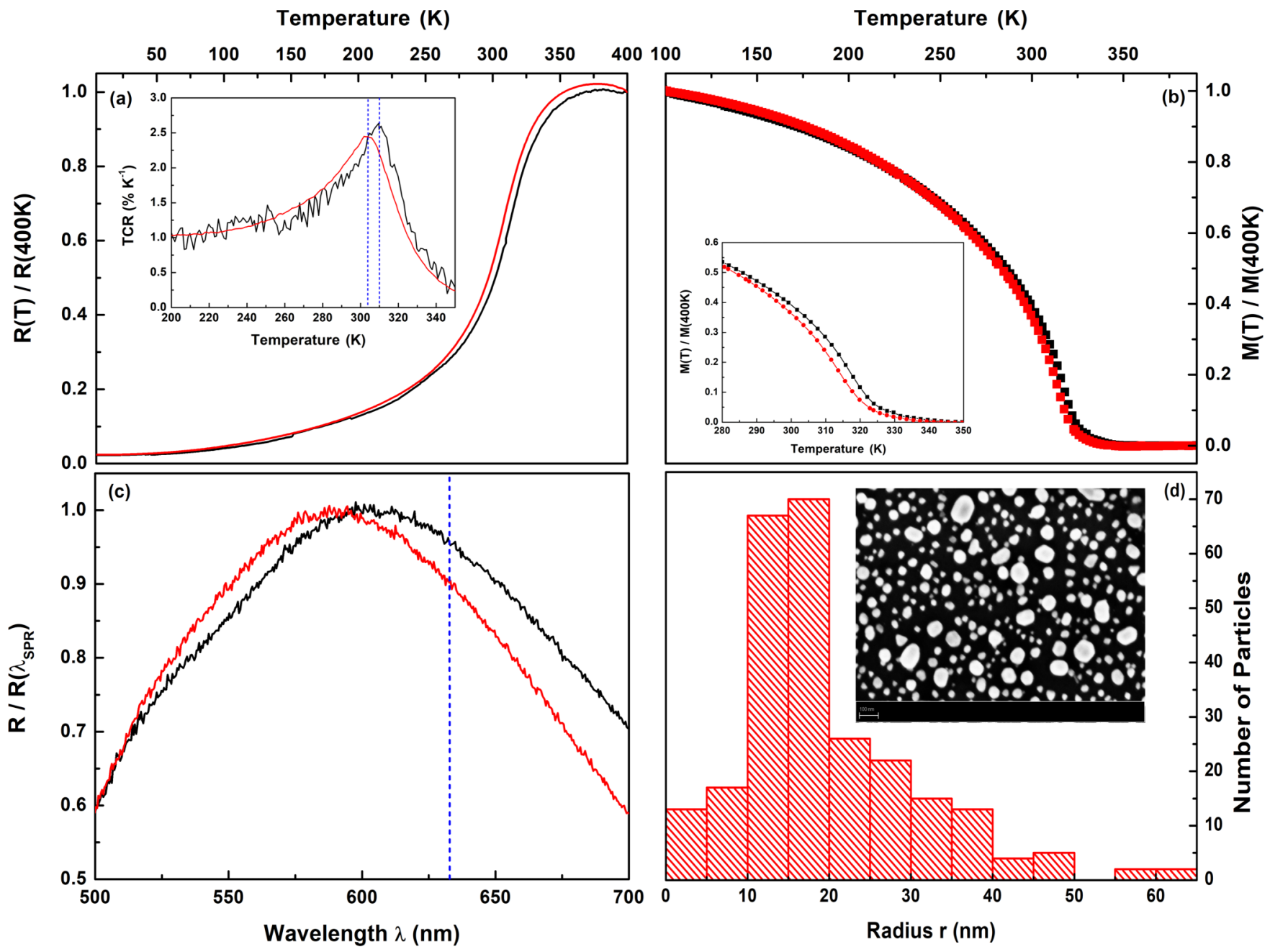

FIG. 1. (a) Electrical and (b) magnetic characterization of the ultrathin LSMO film $(d=12$ u.c.). Slight changes of the magnetic and electric transition temperatures infer negligible deoxygenation effects (black: LSMO; red: Au/LSMO). Reflectance measurements (c) provide a SPR wavelength $\lambda_{\mathrm{SPR}}=606 \mathrm{~nm}$ for LCMO (black) and $\lambda_{\mathrm{SPR}}=592 \mathrm{~nm}$ for LSMO (red), respectively. SEM pictures of an exemplary $\mathrm{Au} /$ manganite structure show the formation of Au-NPs with a mean particle size, $d_{\mathrm{NP}} \approx 15-16 \mathrm{~nm}$, obtained from the particle size distribution (d).

the orthorhombic structure [33]. However, since the bending mode and the JT modes play an important role in the metalinsulator transition $[30,56]$ and the rotational mode is sensitive to structural changes, e.g., octahedral tilting due to doping [57], the focus in the following discussion will be on these four modes. A direct comparison of the "bulk" Raman spectra and the surface-enhanced Raman (SER) spectra of LCMO reveals only slight differences, i.e., an enhancement of the spectra and a peak shift of the rotational mode as well as of the JT stretching modes. Furthermore, an additional mode around $568 \mathrm{~cm}^{-1}$ is observable [see Fig. 2(c)]. A simple approach to quantify the enhancement is to calculate the intensity contrast, $C=I_{\mathrm{SERS}} / I_{\mathrm{RS}}$, with $I_{\mathrm{SERS}}$ as SER and $I_{\mathrm{RS}}$ as normal Raman intensity. The obtained intensity contrasts are summarized in Table I. Remarkably, both stretching modes have a different sign of peak shift $\Delta \omega=\omega_{\text {SERS }}-\omega_{\text {RS }}$ : a blueshift for the as-JT mode and a redshift for the s-JT mode.

In contrast to LCMO, the most prominent features in the "bulk" Raman spectra of LSMO [Fig. 2(b)] are the rotational mode at $178 \mathrm{~cm}^{-1}\left(A_{1 \mathrm{~g}}\right)$ and the bending mode at $422 \mathrm{~cm}^{-1}$
$\left(E_{\mathrm{g}}\right)$, whose positions are consistent with previous observations [32,58] and lattice dynamical calculations [35]. Additionally, weak contributions of the "orthorhombic" modes at 331 and $396 \mathrm{~cm}^{-1}$ and the JT modes at 470 and $630 \mathrm{~cm}^{-1}$ can be seen. Since these modes are forbidden in the rhombohedral structure of LSMO [35,36], we assign their appearance in the bulk spectra to a small orthorhombic distortion at the film/substrate interface, which is in agreement with previous results $[45,59,60]$. The huge strain induced by the large mismatch between the $\mathrm{MgO}(100)$ substrate $\left(a_{\mathrm{MgO}}=4.209 \AA\right.$ [47]) and LSMO ( $a_{\text {bulk }}=3.889 \AA$ [46]) is released by the formation of misfit dislocations, located within the first $\approx 2 \mathrm{~nm}$ from the interface [47,61]. After this defect- and strain-rich region, the film grows unstrained and almost defect free [61] with a pseudocubic lattice constant, $a_{\text {film }}=3.882 \AA$, which is almost identical to the bulk value. These misfit dislocations are responsible for the observed orthorhombic distortions. Additionally, a broad hump centered around $515 \mathrm{~cm}^{-1}$ is clearly seen in the bulk spectra of LSMO and corresponds to an collective plasmalike excitation [62]. The SER spectra 

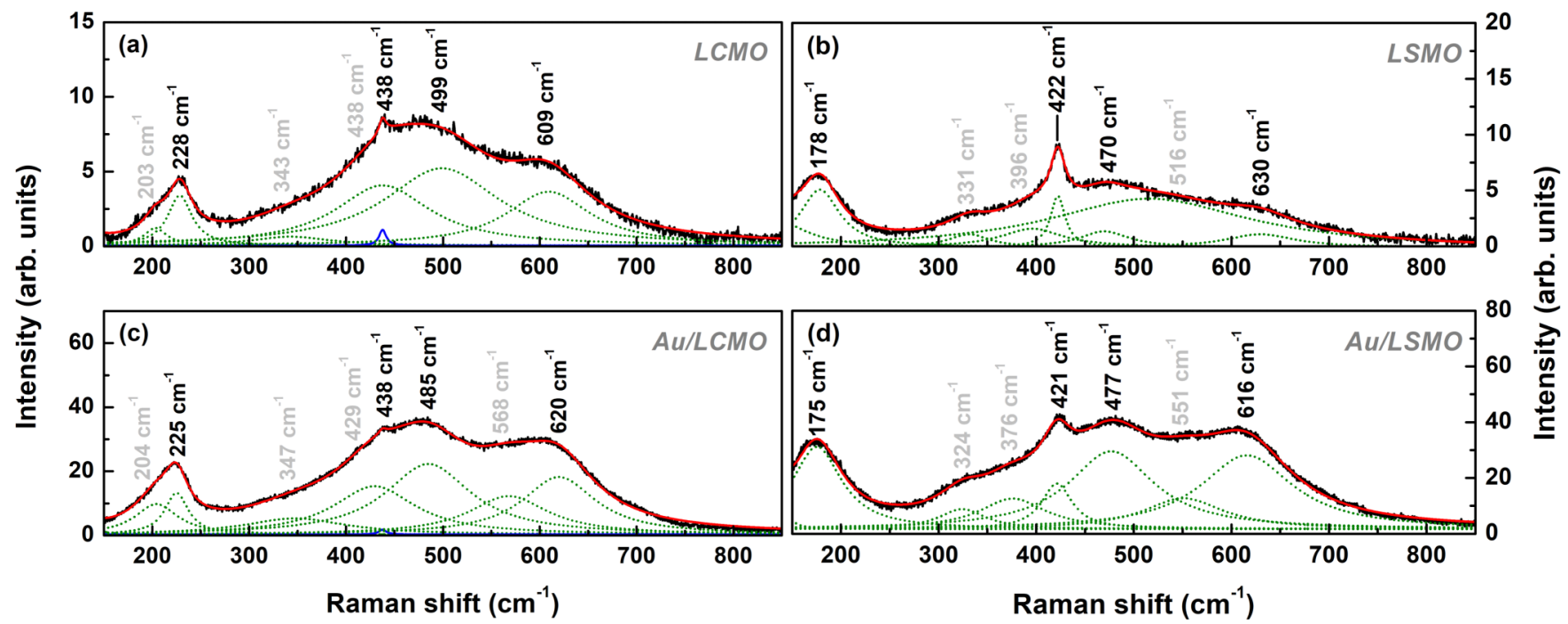

FIG. 2. Comparison between the "bulk" Raman spectra of (a) LCMO and (b) LSMO and (c), (d) their corresponding SER spectra. For LCMO (c), the Au-NP deposition results in an enhancement of the Raman spectra and an additional mode around $568 \mathrm{~cm}^{-1}$. The SER spectra of LSMO (d) show an moderate enhancement of the rotational and bending mode as well as the strong enhancement of the JT modes at 477 and $616 \mathrm{~cm}^{-1}$, indicating a Jahn-Teller distorted surface structure.

of LSMO, shown in Fig. 2(d), reveal an additional mode at $551 \mathrm{~cm}^{-1}$ and a strong enhancement of the JT modes at 477 and $616 \mathrm{~cm}^{-1}$, compared to the relatively weak enhancement of the rotational and bending mode (see Table I). However, this strong intensity enhancement cannot be attributed to an enhancement of the film/substrate interface, since the electric field of the surface plasmon decays strongly within the film. Considering the thickness of the film, $d_{\text {film }}=89 \mathrm{~nm}$, and the distance dependence of the plasmonic enhancement [38], $I \propto 1 / r^{-10}$, the intensity enhancement at the interface would be of the order of $10^{-8}$ compared to that of the surface. Hence, the observed enhancement of the JT modes in the SER spectra of LSMO is certainly a surface phenomenon. This is further supported by the observed peak shift of the JT modes. The absence of bonding partners at the surface causes a deviation of the $\mathrm{Mn}-\mathrm{O}$ bond length $d_{\mathrm{Mn}-\mathrm{O}}$ [14], which will be reflected in the vibrational frequency. However, the redshift of the s-JT mode in LCMO indicates a smaller $d_{\mathrm{Mn}-\mathrm{O}}$, but the blueshift of the as-JT mode a larger $d_{\mathrm{Mn}-\mathrm{O}}$ [63]. Similar considerations apply to LSMO, but here the JT modes are related to the film/substrate interface and not the bulk structure. The different sign of the peak shift suggests an asymmetric change of $d_{\mathrm{Mn}-\mathrm{O}}$ favoring the asymmetric stretching of the in-plane oxygen in LCMO, but the symmetric stretching in LSMO.

The appearance of the JT-like modes at the LSMO surface could be caused by different processes: A charge transfer from the bulk to the surface [11] would lead to the formation of an insulating $\mathrm{Mn}^{3+}$-rich layer at the surface

TABLE I. Intensity contrasts obtained for the different modes of LCMO and LSMO.

\begin{tabular}{lccrr}
\hline \hline & Rotational & Bending & as-JT & s-JT \\
\hline LCMO & 3.75 & 1.5 & 3.15 & 4.34 \\
LSMO & 7.35 & 7 & 34.64 & 30.52 \\
\hline \hline
\end{tabular}

in doped manganites with a hole concentration $x=0.3$. As a consequence, the crystal structure changes from the rhombohedral $(R \overline{3} c)$ in the bulk to the orthorhombic (Pnma) structure at the surface [14]. Since the JT modes are allowed in this structure, the enrichment of the JT-active $\mathrm{Mn}^{3+}$ ion and the lowering of the symmetry should lead to the appearance of the JT modes within the insulating surface layer. Such a symmetry lowering cannot occur in an orthorhombic system, since it already resides in the low-symmetric stable structure, predicted by the tolerance factor for perovskite oxides [64]. This is supported by theoretical calculations on orthorhombic $\mathrm{LaMnO}_{3}$ [8] and $\mathrm{CaMnO}_{3}[9,10]$ and in good agreement with the observed enhancement in our SER spectra of LCMO. Chemical effects such as segregation of the dopant $[23-25,28]$ are well known for doped manganites. However, since segregation is favored by a low oxygen partial pressure [28], these effects should be suppressed due to the high $p\left(\mathrm{O}_{2}\right)$ provided by the MAD. An enrichment of $\mathrm{Sr} / \mathrm{Ca}$ at the surface would further result in a higher effective doping at the surface, leading thus to a reduction of the JT modes [65] and not an enhancement. Another possible explanation for the appearance of the JT-like modes at the LSMO surface is the Au-NP deposition itself. X-ray absorption spectroscopy on $\operatorname{LSMO}(d=40 \mathrm{~nm})$ with an Au-capping layer $(d=2 \mathrm{~nm})$ revealed the presence of $\mathrm{Mn}^{2+}$ at the interface between the film and capping layer [66]. Additionally, the deposition of $\mathrm{Au}-\mathrm{NPs}$ onto the surface creates an interface between the gold and the manganite surface, which could cause the observed symmetry breaking. However, the formation of $\mathrm{Mn}^{2+}$ as well as the artificially created symmetry breaking would impact the $\mathrm{Mn}^{3+} / \mathrm{Mn}^{4+}$ ratio and thus result in a decrease of the transition temperature. As mentioned before, even an ultrathin film does not show any significant changes of its properties after the Au-NP deposition. Therefore, the formation of $\mathrm{Mn}^{2+}$ or an artificially induced symmetry breaking caused by the Au-NP deposition is unlikely. Hence, the JT-like modes have 


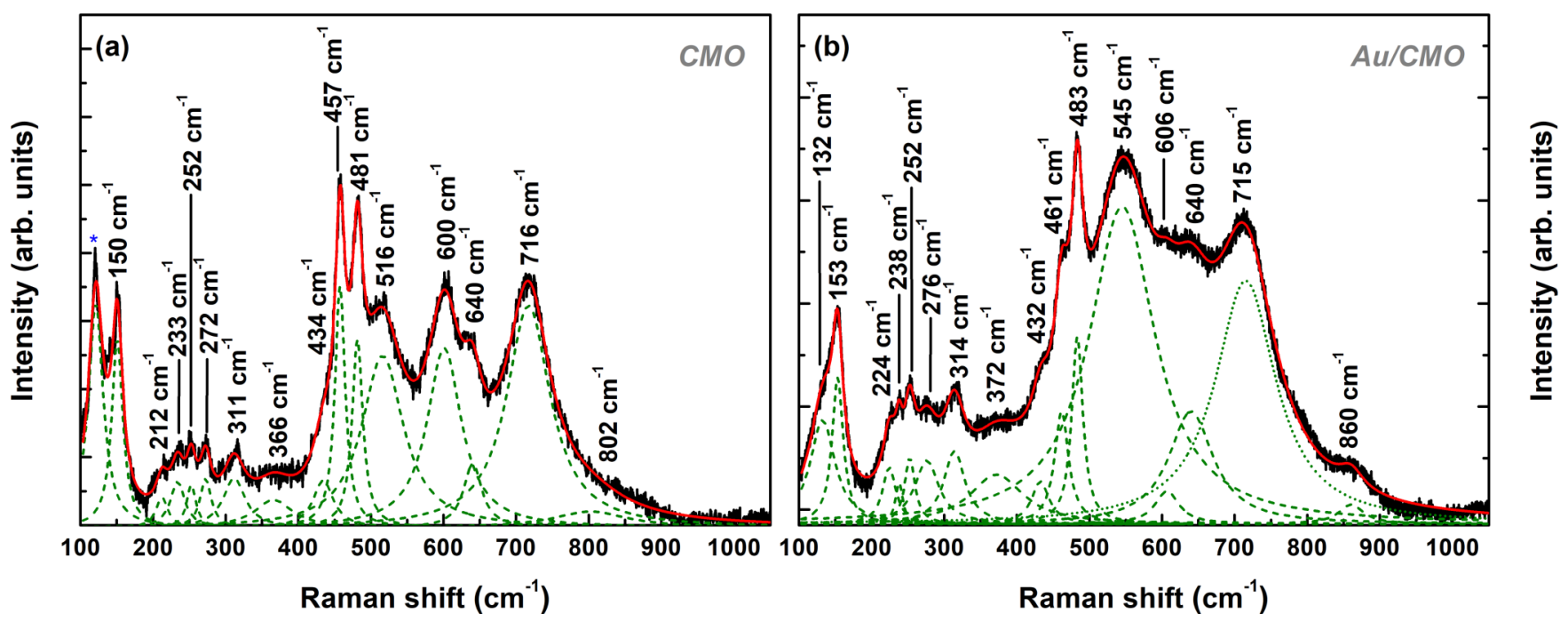

FIG. 3. (a) Bulk and (b) SER spectra of $\mathrm{CaMnO}_{3}$ reveal no Jahn-Teller-related Raman modes, supporting the authenticity of our LSMO spectra and the appearance of the Jahn-Teller stretching mode.

to be an intrinsic electronic surface phenomenon or they are related to the Au-NPs themselves.

To verify the authenticity of the appearing JT-like modes at the LSMO surface and to exclude effects caused by the Au-NPs themselves, we have grown orthorhombic $\mathrm{CaMnO}_{3}$ thin films on the lattice-matched $\mathrm{LaAlO}_{3}$ (100) substrates (see Fig. 1.5 in SM I [45] for plasmonic characterization). $\mathrm{CaMnO}_{3}$ shows a 60 times smaller JT distortion than the orthorhombic $\mathrm{LaMnO}_{3}$ and does not feature any Jahn-Tellerrelated Raman modes [Fig. 3(a)] [67]. Slab calculations of the $\mathrm{CaMnO}_{3}$ surface predict only a possible change of the magnetic order $[9,10]$, but no electronic or structural reconstruction, which could cause a stronger JT distortion. As one can see in Fig. 3(b), there are no additional JT-like modes present in the SER spectra of $\mathrm{CaMnO}_{3}$. This verifies the authenticity of the observed JT-like modes in the SER spectra of LSMO and excludes their appearance caused by the AuNPs themselves [68]. However, as in LCMO and LSMO, an additional mode at $545 \mathrm{~cm}^{-1}$ is clearly visible in the SER spectra of $\mathrm{CaMnO}_{3}$ and likely will be of similar origin as the additional surface mode in LCMO and LSMO.

Hence, we can conclude that the strong enhancement of the JT-like modes has to be an intrinsic surface phenomenon of the LSMO surface and that they can indeed be assigned to the JT stretching vibrations, which are inherent to the orthorhombic (Pnma) structure of the lightly doped manganites. Note that we assume a $\mathrm{MnO}_{2}$-terminated surface of our manganite films as observed in previous works [16,17]. But, since the surface termination of the studied films is unknown, one has to keep in mind that a different termination of the manganite surface could lead to an alternative surface reconstruction and thus to the suppression of the JT modes. Nonetheless, we can experimentally confirm herewith the theoretically predicted symmetry lowering at the manganite surface and the formation of an electron-rich JT distorted surface layer in the ferromagnetic metallic LSMO as well as the electron enrichment at the surface of the orthorhombic LCMO.

To model the surface structure of LSMO and LCMO, we compared the intensity contrast of the different modes (see
Table I). By keeping in mind the strong distance dependence of the plasmonic enhancement [38], the significantly higher contrast of the JT modes in comparison to the bending mode can be directly linked to a stronger surface contribution of them. Hence, the bending mode can be considered as a bulk mode and can be taken to estimate the thickness of the JahnTeller distorted surface layer. The treatment as a bulk mode is further supported by its negligible peak shift, which would be expected from an undisturbed structure. Using the simplified sphere model and the $|E|^{4}$ approximation, the SER intensity scales with [38]

$$
I_{\text {SERS }} \sim[(1+r / a)]^{-10},
$$

with $I_{\text {SERS }}$ the SER intensity of the Raman mode, $a$ the radius of curvature of the field enhancing feature of the metallic surface, and $r$ the distance from the metallic surface to the adsorbate. Assuming the radius of curvature is given by the mean particle size $d_{\mathrm{NP}} \approx 16 \mathrm{~nm}$, Eq. (1) yields the distance dependence shown in Fig. 4. Since we consider the bending mode as a bulk mode, the ratio $\eta=C_{\mathrm{bend}} / C_{\mathrm{JT}}$ represents the weakening of the plasmonic enhancement within the whole distorted surface layer. To estimate the thickness of the surface layer, one compares the determined ratio with the distance dependence in Fig. 4. For LSMO, the determined ratio $\eta \approx 7 / 31$ yields a thickness of the surface layer, $d_{\mathrm{JT}} \approx 7$ u.c. $\approx 2.8 \mathrm{~nm}$. Similar considerations for the modes of LCMO yield a significantly smaller thickness $d_{\mathrm{JT}} \approx 4-5$ u.c. $\approx 1.7-2.0 \mathrm{~nm}$. Both values correspond well to the thicknesses of the insulating antiferromagnetic surface layer obtained from XMCD [20] and XRMS [22]. The inset in Fig. 4 summarizes the proposed model of the manganite surface. Note that the intensity contrast of the rotational mode in LSMO is only slightly higher than the contrast of the bending mode, indicating a rather bulk-related than surface-related nature. In LCMO, however, the intensity contrast is similar to those of the JT modes and thus will be associated with the surface structure. As mentioned before, this mode is sensitive to the tilting of the $\mathrm{MnO}_{6}$ octahedron, so that its blueshift suggests a change of the octahedral tilting near the surface. However, both manganite systems show 

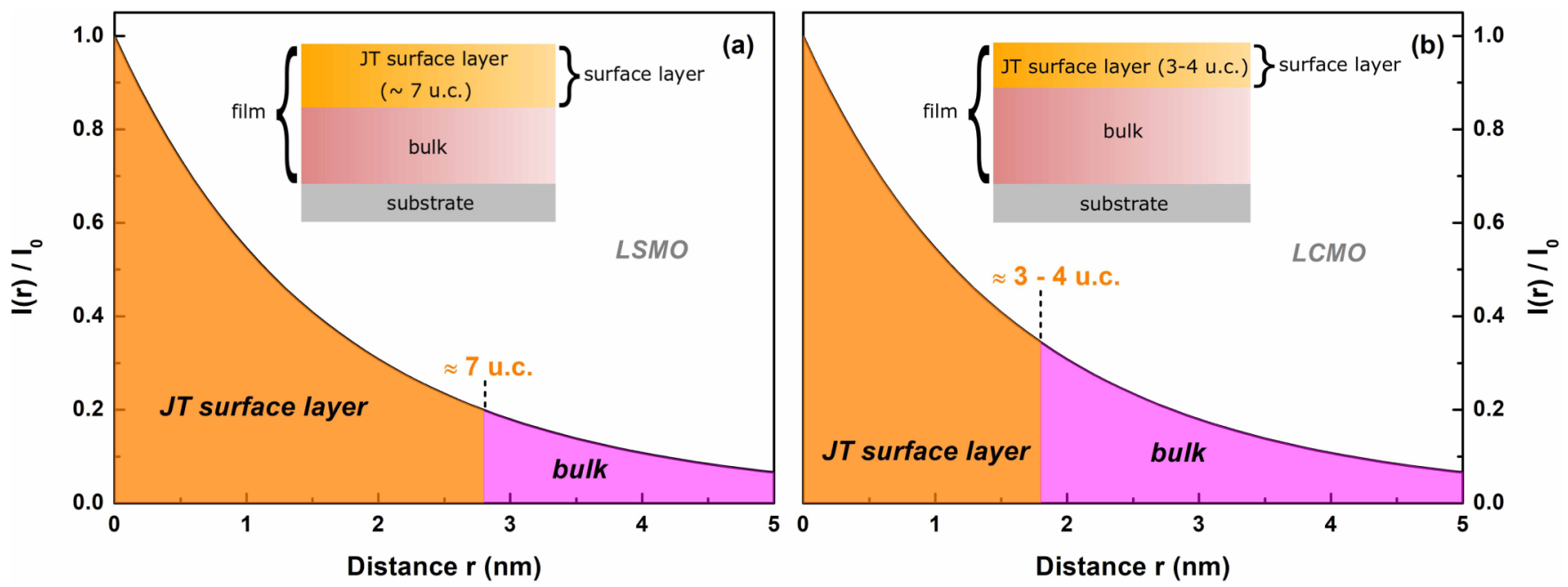

FIG. 4. The modeled (a) LSMO and (b) LCMO surface structure, determined from the distance dependence of the electromagnetic enhancement due to the plasmonic excitation in the Au-NPs.

a comparable peak shift and therefore a similar octahedral tilting. Considering that a structural transition occurs at the LSMO surface, this is quite remarkable and, in combination with the slightly higher contrast of the rotational mode, hints at the presence of an intermediate layer, which accommodates the structural transition at the surface.

Finally, we want to discuss the assignment of the additional mode around $550-570 \mathrm{~cm}^{-1}$. Compared to theoretical calculations $[33,67]$, the closest mode would be the out-of-phase stretching mode at $536 \mathrm{~cm}^{-1}$ in $\mathrm{CaMnO}_{3}$ and $575 \mathrm{~cm}^{-1}$ in $\mathrm{LaMnO}_{3}$ of $B_{1 \mathrm{~g}}$ symmetry, respectively. Although, our backscattering geometry only allows the detection of the $A_{1 \mathrm{~g}}$ and $B_{2 \mathrm{~g}}$ modes, one can imagine that the symmetry breaking at the surface and the rearrangement of the $\mathrm{MnO}_{6}$ octahedron could result in a structural distortion at the surface allowing the observation of $B_{1 \mathrm{~g}}$ modes. Further studies, especially from the theoretical point of view, could give insight into the origin of the additional mode and our proposed assignment.

\section{CONCLUSION}

We have studied the surface structure of the mixed-valence perovskite manganites by means of SERS. The SER spectra reveal an electron-rich surface structure, which is manifested by the dominance of the JT stretching modes. The strong enhancement of the JT modes in LSMO was attributed to a structural transition to the orthorhombic (Pnma) structure at the surface, which results in a unique surface structure. This is a direct experimental confirmation of the theoretically predicted surface of the doped manganite with $x=0.3$. The authenticity of our SERS results obtained for the rhombohedral LSMO is supported by the SER spectra of orthorhombic $\mathrm{LCMO}$ and CMO.

\section{ACKNOWLEDGMENT}

The authors acknowledge financial support from the Deutsche Forschungsgemeinschaft (DFG) via SFB 1073 (TP B04, B01 and A02).
[1] E. Dagotto, M. Cardona, P. Fulde, K. von Klitzing, R. Merlin, H.-J. Queisser, and H. Störmer, Nanoscale Phase Separation and Colossal Magnetoresistance, Springer Series in Solid-State Sciences Vol. 136 (Springer, Berlin, 2003).

[2] V. Moshnyaga and K. Samwer, Electron-lattice correlations and phase transitions in CMR manganites, Ann. Phys. 523, 652 (2011).

[3] W. E. Pickett and D. J. Singh, Transport and fermiology of the ferromagnetic phase of $\mathrm{La}_{0.7} A_{0.3} \mathrm{MnO}_{3}(A=\mathrm{Ca}, \mathrm{Sr}, \mathrm{Ba})$, J. Magn. Magn. Mater. 172, 237 (1997).

[4] C. H. Kim, G. Qi, K. Dahlberg, and W. Li, Strontium-doped perovskites rival platinum catalysts for treating $\mathrm{NO}_{x}$ in simulated diesel exhaust, Science 327, 1624 (2010).

[5] M. Viret, J. Nassar, M. Drouet, J. Contour, C. Fermon, and A. Fert, Spin polarised tunnelling as a probe of half metallic ferromagnetism in mixed-valence manganites, J. Magn. Magn. Mater. 198-199, 1 (1999).
[6] J. Suntivich, H. A. Gasteiger, N. Yabuuchi, H. Nakanishi, J. B. Goodenough, and Y. Shao-Horn, Design principles for oxygenreduction activity on perovskite oxide catalysts for fuel cells and metal-air batteries, Nat. Chem. 3, 546 (2011).

[7] R. A. Evarestov, E. A. Kotomin, Y. A. Mastrikov, D. Gryaznov, E. Heifets, and J. Maier, Comparative density-functional LCAO and plane-wave calculations of $\mathrm{LaMnO}_{3}$ surfaces, Phys. Rev. B 72, 214411 (2005).

[8] F. L. Tang, M. Huang, W. J. Lu, and W. Y. Yu, Structural relaxation and Jahn-Teller distortion of $\mathrm{LaMnO}_{3}$ (001) surface, Surf. Sci. 603, 949 (2009).

[9] A. Filippetti and W. E. Pickett, Magnetic Reconstruction at the (001) $\mathrm{CaMnO}_{3}$ Surface, Phys. Rev. Lett. 83, 4184 (1999).

[10] S. Keshavarz, Y. O. Kvashnin, D. C. M. Rodrigues, M. Pereiro, I. Di Marco, C. Autieri, L. Nordström, I. V. Solovyev, B. Sanyal, and O. Eriksson, Exchange interactions of $\mathrm{CaMnO}_{3}$ in the bulk and at the surface, Phys. Rev. B 95, 115120 (2017). 
[11] M. J. Calderón, L. Brey, and F. Guinea, Surface electronic structure and magnetic properties of doped manganites, Phys. Rev. B 60, 6698 (1999).

[12] A. Filippetti and W. E. Pickett, Double-exchange-driven spin pairing at the (001) surface of manganites, Phys. Rev. B 62, 11571 (2000).

[13] H. Zenia, G. A. Gehring, G. Banach, and W. M. Temmerman, Electronic and magnetic properties of the (001) surface of holedoped manganites, Phys. Rev. B 71, 024416 (2005).

[14] J. M. Pruneda, V. Ferrari, R. Rurali, P. B. Littlewood, N. A. Spaldin, and E. Artacho, Ferrodistortive Instability at the (001) Surface of Half-Metallic Manganites, Phys. Rev. Lett. 99, 226101 (2007).

[15] Y. Mastrikov, E. Heifets, E. A. Kotomin, and J. Maier, Atomic, electronic and thermodynamic properties of cubic and orthorhombic $\mathrm{LaMnO}_{3}$ surfaces, Surf. Sci. 603, 326 (2009).

[16] M. Yoshimoto, H. Maruta, T. Ohnishi, K. Sasaki, and H. Koinuma, In situ determination of the terminating layer of $\mathrm{La}_{0.7} \mathrm{Sr}_{0.3} \mathrm{MnO}_{3}$ thin films using coaxial impact-collision ion scattering spectroscopy, Appl. Phys. Lett. 73, 187 (1998).

[17] J. Choi, J. Zhang, S.-H. Liou, P. A. Dowben, and E. W. Plummer, Surfaces of the perovskite manganites $\mathrm{La}_{1-x} \mathrm{Ca}_{x} \mathrm{MnO}_{3}$, Phys. Rev. B 59, 13453 (1999).

[18] L. Abad, B. Martínez, and L. Balcells, Surface behavior of $\mathrm{La}_{0.7} \mathrm{Ca}_{0.3} \mathrm{MnO}_{3}$ epitaxial thin films, Appl. Phys. Lett. 87, 212502 (2005).

[19] J.-O. Krisponeit, C. Kalkert, B. Damaschke, V. Moshnyaga, and K. Samwer, Nanoscale resistance switching in manganite thin films: Sharp voltage threshold and pulse-width dependence, Phys. Rev. B 82, 144440 (2010).

[20] S. Valencia, A. Gaupp, W. Gudat, L. Abad, L. Balcells, and B. Martínez, Surface degradation of magnetic properties in manganite thin films proved with magneto-optical techniques in reflection geometry, Appl. Phys. Lett. 90, 252509 (2007).

[21] J. W. Freeland, K. E. Gray, L. Ozyuzer, P. Berghuis, E. Badica, J. Kavich, H. Zheng, and J. F. Mitchell, Full bulk spin polarization and intrinsic tunnel barriers at the surface of layered manganites, Nat. Mater. 4, 62 (2004).

[22] J. W. Freeland, J. J. Kavich, K. E. Gray, L. Ozyuzer, H. Zheng, J. F. Mitchell, M. P. Warusawithana, P. Ryan, X. Zhai, R. H. Kodama, and J. N. Eckstein, Suppressed magnetization at the surfaces and interfaces of ferromagnetic metallic manganites, J. Phys.: Condens. Matter 19, 315210 (2007).

[23] R. Bertacco, J. Contour, A. Barthélemy, and J. Olivier, Evidence for strontium segregation in $\mathrm{La}_{0.7} \mathrm{Sr}_{0.3} \mathrm{MnO}_{3}$ thin films grown by pulsed laser deposition: Consequences for tunnelling junctions, Surf. Sci. 511, 366 (2002).

[24] W. Lee, J. W. Han, Y. Chen, Z. Cai, and B. Yildiz, Cation size mismatch and charge interactions drive dopant segregation at the surfaces of manganite perovskites, J. Am. Chem. Soc. 135, 7909 (2013).

[25] H. Dulli, P. A. Dowben, S.-H. Liou, and E. W. Plummer, Surface segregation and restructuring of colossal-magnetoresistant manganese perovskites $\mathrm{La}_{0.65} \mathrm{Sr}_{0.35} \mathrm{MnO}_{3}$, Phys. Rev. B 62, R14629 (2000).

[26] C. N. Borca, D. Ristoiu, Q. L. Xu, S.-H. Liou, S. Adenwalla, and P. A. Dowben, The surface terminal layer and composition of the colossal magnetoresistance perovskite: $\mathrm{La}_{0.65} \mathrm{~Pb}_{0.35} \mathrm{MnO}_{3}$, J. Appl. Phys. 87, 6104 (2000).
[27] M. P. de Jong, V. A. Dediu, C. Taliani, and W. R. Salaneck, Electronic structure of $\mathrm{La}_{0.7} \mathrm{Sr}_{0.3} \mathrm{MnO}_{3}$ thin films for hybrid organic/inorganic spintronics applications, J. Appl. Phys. 94, 7292 (2003).

[28] T. T. Fister, D. D. Fong, J. A. Eastman, P. M. Baldo, M. J Highland, P. H. Fuoss, K. R. Balasubramaniam, J. C. Meador, and P. A. Salvador, In situ characterization of strontium surface segregation in epitaxial $\mathrm{La}_{0.7} \mathrm{Sr}_{0.3} \mathrm{MnO}_{3}$ thin films as a function of oxygen partial pressure, Appl. Phys. Lett. 93, 151904 (2008).

[29] T. P. Devereaux and R. Hackl, Inelastic light scattering from correlated electrons, Rev. Mod. Phys. 79, 175 (2007).

[30] M. N. Iliev, M. V. Abrashev, V. N. Popov, and V. G. Hadjiev, Role of Jahn-Teller disorder in Raman scattering of mixedvalence manganites, Phys. Rev. B 67, 212301 (2003).

[31] V. Moshnyaga, A. Belenchuk, S. Hühn, C. Kalkert, M Jungbauer, O. I. Lebedev, S. Merten, K.-Y. Choi, P. Lemmens, B. Damaschke, and K. Samwer, Intrinsic antiferromagnetic coupling underlies colossal magnetoresistance effect: Role of correlated polarons, Phys. Rev. B 89, 024420 (2014).

[32] E. Granado, N. O. Moreno, A. García, J. A. Sanjurjo, C. Rettori, I. Torriani, S. B. Oseroff, J. J. Neumeier, K. J. McClellan, S.-W. Cheong, and Y. Tokura, Raman scattering in CMR manganites, Mater. Sci. Forum 302-303, 134 (1999).

[33] M. N. Iliev, M. V. Abrashev, H.-G. Lee, V. N. Popov, Y. Y. Sun, C. Thomsen, R. L. Meng, and C. W. Chu, Raman spectroscopy of orthorhombic perovskitelike $\mathrm{YMnO}_{3}$ and $\mathrm{LaMnO}_{3}$, Phys. Rev. B 57, 2872 (1998).

[34] M. N. Iliev, M. V. Abrashev, J. Laverdière, S. Jandl, M. M. Gospodinov, Y.-Q. Wang, and Y.-Y. Sun, Distortion-dependent Raman spectra and mode mixing in $R \mathrm{MnO}_{3}$ perovskites $(R=$ La, Pr, Nd, Sm, Eu, Gd, Tb, Dy, Ho, Y), Phys. Rev. B 73, 064302 (2006).

[35] M. Talati and P. K. Jha, Phonons and Jahn-Teller distortion in manganites, J. Mol. Struct. 838, 227 (2007).

[36] M. N. Iliev and M. V. Abrashev, Raman phonons and Raman Jahn-Teller bands in perovskite-like manganites, J. Raman Spectrosc. 32, 805 (2001).

[37] S. Schlücker, Surface-enhanced Raman spectroscopy: Concepts and chemical applications, Angew. Chem., Int. Ed. 53, 4756 (2014).

[38] B. J. Kennedy, S. Spaeth, M. Dickey, and K. T. Carron, Determination of the distance dependence and experimental effects for modified SERS substrates based on self-assembled monolayers formed using alkanethiols, J. Phys. Chem. B 103, 3640 (1999).

[39] S. S. Masango, R. A. Hackler, N. Large, A.-I. Henry, M. O. McAnally, G. C. Schatz, P. C. Stair, and R. P. van Duyne, High-resolution distance dependence study of surfaceenhanced Raman scattering enabled by atomic layer deposition, Nano Lett. 16, 4251 (2016)

[40] L. Yang, M. Gong, X. Jiang, Di Yin, X. Qin, B. Zhao, and W. Ruan, Investigation on SERS of different phase structure $\mathrm{TiO}_{2}$ nanoparticles, J. Raman Spectrosc. 46, 287 (2015).

[41] C.-T. Ko, P.-S. Yang, Y.-Y. Han, W.-C. Wang, J.-J. Huang, Y.-H. Lee, Y.-J. Tsai, J. Shieh, and M.-J. Chen, Atomic-layerdeposited silver and dielectric nanostructures for plasmonic enhancement of Raman scattering from nanoscale ultrathin films, Nanotechnology 26, 265702 (2015).

[42] O. Prakash, S. Kumar, P. Singh, V. Deckert, S. Chatterjee, A. K. Ghosh, and R. K. Singh, Surface-enhanced Raman scattering 
characteristics of $\mathrm{CuO}: \mathrm{Mn} / \mathrm{Ag}$ heterojunction probed by methyl orange: Effect of $\mathrm{Mn}^{2+}$ doping, J. Raman Spectrosc. 47, 813 (2016).

[43] C. Meyer, S. Hühn, M. Jungbauer, S. Merten, B. Damaschke, K. Samwer, and V. Moshnyaga, Tip-enhanced Raman spectroscopy (TERS) on double perovskite $\mathrm{La}_{2} \mathrm{CoMnO}_{6}$ thin films: Field enhancement and depolarization effects, J. Raman Spectrosc. 48, 46 (2017).

[44] V. Moshnyaga, I. Khoroshun, A. Sidorenko, P. Petrenko, A. Weidinger, M. Zeitler, B. Rauschenbach, R. Tidecks, and K. Samwer, Preparation of rare-earth manganite-oxide thin films by metalorganic aerosol deposition technique, Appl. Phys. Lett. 74, 2842 (1999).

[45] See Supplemental Material at http://link.aps.org/supplemental/ 10.1103/PhysRevMaterials.3.060401 for the characterization of the studied thin films, the background substraction procedure of the Raman spectra, and a sketch of the optical reflectance setup.

[46] C. Kwon, M. C. Robson, K.-C. Kim, J. Y. Gu, S. E. Lofland, S. M. Bhagat, Z. Trajanovic, M. Rajeswari, T. Venkatesan, A. R. Kratz, R. D. Gomez, and R. Ramesh, Stress-induced effects in epitaxial $\mathrm{La}_{0.7} \mathrm{Sr}_{0.3} \mathrm{MnO}_{3}$ films, J. Magn. Magn. Mater. 172, 229 (1997).

[47] E. Gommert, H. Cerva, J. Wecker, and K. Samwer, Influence of misfit stress on the magnetoresistive properties of $\mathrm{La}_{0.7} \mathrm{Ca}_{0.3} \mathrm{MnO}_{3-\delta}$ thin films, J. Appl. Phys. 85, 5417 (1999).

[48] D. Petti, A. Stroppa, S. Picozzi, S. Brivio, M. Cantoni, and R. Bertacco, Effect of Au proximity on the LSMO surface: An $a b$ initio study, J. Magn. Magn. Mater. 324, 2659 (2012).

[49] D. Petti, R. Bertacco, S. Brivio, M. Cantoni, A. Cattoni, and F. Ciccacci, X-ray photoemission study of the $\mathrm{Au} / \mathrm{La}_{0.67} \mathrm{Sr}_{0.33} \mathrm{MnO}_{3}$ interface formation, J. Appl. Phys. 103, 044903 (2008).

[50] S. Brivio, C. Magen, A. A. Sidorenko, D. Petti, M. Cantoni, M. Finazzi, F. Ciccacci, R. De Renzi, M. Varela, S. Picozzi, and R. Bertacco, Effects of Au nanoparticles on the magnetic and transport properties of $\mathrm{La}_{0.67} \mathrm{Sr}_{0.33} \mathrm{MnO}_{3}$ ultrathin layers, Phys. Rev. B 81, 094410 (2010).

[51] J. Dho, N. H. Hur, I. S. Kim, and Y. K. Park, Oxygen pressure and thickness dependent lattice strain in $\mathrm{La}_{0.7} \mathrm{Sr}_{0.3} \mathrm{MnO}_{3}$ films, Appl. Phys. Lett. 94, 7670 (2003).

[52] M. Jungbauer, S. Hühn, R. Egoavil, H. Tan, J. Verbeeck, G. van Tendeloo, and V. Moshnyaga, Atomic layer epitaxy of Ruddlesden-Popper $\mathrm{SrO}\left(\mathrm{SrTiO}_{3}\right)_{n}$ films by means of metalorganic aerosol deposition, Appl. Phys. Lett. 105, 251603 (2014).

[53] R. Bertacco, S. Brivio, M. Cantoni, A. Cattoni, D. Petti, M. Finazzi, F. Ciccacci, A. A. Sidorenko, M. Ghidini, G. Allodi, and R. De Renzi, Proximity effects induced by a gold layer on $\mathrm{La}_{0.67} \mathrm{Sr}_{0.33} \mathrm{MnO}_{3}$ thin films, Appl. Phys. Lett. 91, 102506 (2007).

[54] E. N. Aybeke, Y. Lacroute, C. Elie-Caille, A. Bouhelier, E. Bourillot, and E. Lesniewska, Homogeneous large-scale crystalline nanoparticle-covered substrate with high SERS performance, Nanotechnology 26, 245302 (2015).

[55] K. Huang, W. Pan, J. F. Zhu, J. C. Li, N. Gao, C. Liu, L. Ji, E. T. $\mathrm{Yu}$, and J. Y. Kang, Asymmetric light reflectance from metal nanoparticle arrays on dielectric surfaces, Sci. Rep. 5, 18331 (2015).

[56] M. V. Abrashev, V. G. Avanov, M. N. Iliev, R. A. Chakalov, R. I. Chakalova, and C. Thomsen, Raman study of the variations of the Jahn-Teller distortions through the metal-insulator transition in magnetoresistive $\mathrm{La}_{0.7} \mathrm{Ca}_{0.3} \mathrm{MnO}_{3}$ thin films, Phys. Status Solidi B 215, 631 (1999).

[57] V. A. Amelitchev, B. Güttler, O. Y. Gorbenko, A. R. Kaul, A. A. Bosak, and A. Y. Ganin, Structural and chemical analysis of colossal magnetoresistance manganites by Raman spectrometry, Phys. Rev. B 63, 104430 (2001).

[58] L. Martín-Carrón, A. de Andrés, M. J. Martínez-Lope, M. T. Casais, and J. A. Alonso, Raman phonons as a probe of disorder, fluctuations, and local structure in doped and undoped orthorhombic and rhombohedral manganites, Phys. Rev. B 66, 174303 (2002).

[59] P. Dore, P. Postorino, A. Sacchetti, M. Baldini, R. Giambelluca, M. Angeloni, and G. Balestrino, Raman measurements on thin films of the $\mathrm{La}_{0.7} \mathrm{Sr}_{0.3} \mathrm{MnO}_{3}$ manganite: A probe of substrateinduced effects, Eur. Phys. J. B 48, 255 (2005).

[60] J. Kreisel, G. Lucazeau, C. Dubourdieu, M. Rosina, and F. Weiss, Raman scattering study of $\mathrm{La}_{0.7} \mathrm{Sr}_{0.3} \mathrm{MnO}_{3} / \mathrm{SrTiO}_{3}$ multilayers, J. Phys.: Condens. Matter 14, 5201 (2002).

[61] V. Moshnyaga, L. Sudheendra, O. I. Lebedev, S. A. Köster, K. Gehrke, O. Shapoval, A. Belenchuk, B. Damaschke, G. van Tendeloo, and K. Samwer, A-Site Ordering versus Electronic Inhomogeneity in Colossally Magnetoresistive Manganite Films, Phys. Rev. Lett. 97, 107205 (2006).

[62] P. Björnsson, M. Rübhausen, J. Bäckström, M. Käll, S. Eriksson, J. Eriksen, and L. Börjesson, Lattice and charge excitations in $\mathrm{La}_{1-x} \mathrm{Sr}_{x} \mathrm{MnO}_{3}$, Phys. Rev. B 61, 1193 (2000).

[63] J. M. Hollas, Modern Spectroscopy, 4th ed. (Wiley, Chichester, 2004).

[64] A.-M. Haghiri-Gosnet and J.-P. Renard, CMR manganites: Physics, thin films and devices, J. Phys. D 36, R127 (2003).

[65] E. Liarokapis, T. Leventouri, D. Lampakis, D. Palles, J. J. Neumeier, and D. H. Goodwin, Local lattice distortions and Raman spectra in the $\mathrm{La}_{1-x} \mathrm{Ca}_{x} \mathrm{MnO}_{3}$ system, Phys. Rev. B 60, 12758 (1999).

[66] S. Valencia, L. Peña, Z. Konstantinovic, L. Balcells, R. Galceran, D. Schmitz, F. Sandiumenge, M. Casanove, and B. Martínez, Intrinsic antiferromagnetic/insulating phase at manganite surfaces and interfaces, J. Phys.: Condens. Matter 26, 166001 (2014).

[67] M. V. Abrashev, J. Bäckström, L. Börjesson, V. N. Popov, R. A. Chakalov, N. Kolev, R.-L. Meng, and M. N. Iliev, Raman spectroscopy of $\mathrm{CaMnO}_{3}$ : Mode assignment and relationship between Raman line intensities and structural distortions, Phys. Rev. B 65, 184301 (2002).

[68] One can observe a change in the intensity ratios of the modes, which can be discussed in a picture of a distorted surface structure. Every mode is caused by a specific rotation of the $\mathrm{MnO}_{6}$ octahedron, so that a different intensity ratio gives information about the preferred octahedral tilting at the surface. However, this discussion would be beyond the scope of this Rapid Communication and will be discussed elsewhere. 